DAMPT/93-65 and hep-th/9311147

\title{
Uncertainty Relation in Quantum Mechanics with Quantum Group Symmetry
}

\author{
Achim Kempf* \\ Department of Applied Mathematics \& Theoretical Physics \\ University of Cambridge, Cambridge CB3 9EW, U.K. \\ Email: a.kempf@damtp.cambridge.ac.uk
}

November 1993

\begin{abstract}
We study the commutation relations, uncertainty relations and spectra of position and momentum operators within the framework of quantum group symmetric Heisenberg algebras and their (Bargmann-) Fock representations. As an effect of the underlying noncommutative geometry, a length and a momentum scale appear, leading to the existence of minimal nonzero uncertainties in the positions and momenta. The usual quantum mechanical behaviour is recovered as a limiting case for not too small and not too large distances and momenta.
\end{abstract}

\section{Introduction}

The quantum group $S U_{q}(n)$ that we will use here is a generalisation of the Lie group $S U_{q}(n)$. One recovers the Lie group when the real parameter $q$ tends to 1 . Technically we are dealing with a non-commutative non-cocommutative quasitriangular Hopf algebra. Its dual is a noncommutative generalisation of the function algebra on the group manifold. We can thus consider the quantum group as an example of noncommutative geometry [1, 2, 3, 迎.

It is tempting to examine, whether noncommutative geometry, when introduced into quantum theory, regularises its short distance behaviour. The idea is, not to break symmetries by this regularising procedure, but to (quantum group- ) generalise them instead. One may then even speculate about gravity entering the picture. This

*supported by Studienstiftung des deutschen Volkes, BASF-fellow 
could be in such a way that not only gravity is quantised but also that gravity would feed back to quantum theory by modifying the canonical commutation relations.

We will however for the present confine ourselves to the case of nonrelativistic quantum mechanics. The study of some effects of noncommutative geometry in quantum mechanics was outlined in [5]. Here we cover a more general case and give details and proofs. Our results will support the idea that noncommutative geometry has indeed the potential to regularise ultraviolet and even infrared divergencies in quantum field theories.

\section{$1.1 \quad$ Heisenberg algebra}

In our approach we generate the Heisenberg algebra of $n$ degrees of freedom by mutually adjoint operators $a_{r}$ and $a_{r}^{\dagger},(r=1, \ldots, n)$. This proceeding will automatically supply us with a Hilbert (Fock-) space representation of the Heisenberg algebra. In usual quantum mechanics this is of course equivalent to the use of the hermitean generators $x_{r}$ and $p_{r}$, (which are the well known linear combinations of the former ones) and the representation e.g. on the Hilbert space of square integrable functions.

We will use the quantum group $S U_{q}(n)$ as a 'symmetry' group for nontrivial commutation relations i.e. as linear quantum canonical transformations. Technically the Heisenberg algebra is a $\operatorname{Fun} S U_{q}(n)$-comodule algebra [6]. Arbitrary Hamiltonians can be studied within our framework and they not necessarily have this symmetry.

Explicitely the commutation relations of the following generalised bosonic Heisenberg algebra are conserved under the action of the quantum group $S U_{q}(n)$ :

$$
\begin{aligned}
& a_{i} a_{j}-q a_{j} a_{i}=0 \text { for } i<j \\
& a_{i}^{\dagger} a_{j}^{\dagger}-q a_{j}^{\dagger} a_{i}^{\dagger}=0 \text { for } i>j \\
& a_{i} a_{j}^{\dagger}-q a_{j}^{\dagger} a_{i}=0 \text { for } i \neq j \\
& a_{i} a_{i}^{\dagger}-q^{2} a_{i}^{\dagger} a_{i}=1+\left(q^{2}-1\right) \sum_{j<i} a_{j}^{\dagger} a_{j}
\end{aligned}
$$

Here $i$ runs from 1 to $n$ and $q$ is real. These relations and their fermionic counterpart were derived in the $R$-matrix approach in [6]. As I learned later they had first appeared in a different approach [7]. They are related to the differential calculus on quantum planes [8] and can also be understood as a braided semi direct product construction [9]. Compare also with the different approaches e.g. in [10, 11, 12, 13]. Although quantum groups do in general have more than one free parameter, no further parameters enter in the above commutation relations [14, 15].

\subsection{Bargmann Fock representation}

As usual the Fock space is constructed from a vector $|0\rangle$ with

$$
\langle 0 \mid 0\rangle=1 \quad \text { and } \quad a_{i}|0\rangle=0 \quad \text { for } \quad i=1, \ldots, n
$$


One then obtains for the scalar product:

$$
\left\langle 0\left|\left(a_{n}\right)^{r_{n}} \cdot \ldots \cdot\left(a_{1}\right)^{r_{1}}\left(a_{1}^{\dagger}\right)^{r_{1}} \cdot \ldots \cdot\left(a_{n}^{\dagger}\right)^{r_{n}}\right| 0\right\rangle=\prod_{i=1}^{n}\left[r_{i}\right]_{q} !
$$

with

$$
[r]_{q} !:=[1]_{q} \cdot[2]_{q} \cdot[3]_{q} \cdot \ldots \cdot[r]_{q} \quad \text { and } \quad[p]_{q}:=\frac{q^{2 p}-1}{q^{2}-1}
$$

Thus the scalar product remains positive definite. The Hilbert space $\mathcal{H}$, completed using the induced norm, is as usual isomorphic to the space of square summable series $l^{2}$. The Poincaré series of the $a$ 's and the $a^{\dagger}$ 's remain unchanged.

The usual quantum mechanical programme proved to be possible: The Heisenberg algebra can be represented on a positive definite (Bargmann Fock-) Hilbert space of wave functions and integral kernels like e.g. Green functions can be defined. The one dimensional case had early been studied in [16]. A new 'integral' for calculating the scalar product of two Bargmann Fock wave functions was developed [6, 17], having the samef evaluation procedure in the bosonic as in the fermionic case. Using the undeformed Schrödinger equation, i.e. $i \hbar \partial_{t} \Psi=H \Psi$ with $\partial_{t}$ an ordinary derivative, some dynamical systems were worked out and lead to unitary time evolution [17].

Since the above given commutation relations are respected by formal hermitean conjugation, the natural candidates for position and momentum operators $x \propto a+a^{\dagger}$ and $p \propto i\left(a^{\dagger}-a\right)$ are representable as symmetric operators on a suitable domain. Let us now try to reveal some features of the underlying noncommutative geometry by studying these observables in detail.

\section{Position and momentum operators}

We start with the following ansatz for the position and momentum operators: $(r=1, \ldots, n)$

$$
x_{r}:=L_{r}\left(a_{r}^{\dagger}+a_{r}\right) \quad \text { and } \quad p_{r}:=i K_{r}\left(a_{r}^{\dagger}-a_{r}\right)
$$

Defining their domain $D$ to be

$$
\left.D:=\left\{v \in \mathcal{H}\left|v=\operatorname{Polynomial}\left(a_{1}^{\dagger}, \ldots, a_{n}^{\dagger}\right)\right| 0\right\rangle\right\}
$$

which is dense in $\mathcal{H}$, we insure that all $x_{r}$ and $p_{r}$ are represented as symmetric operators with images that lie in their domain. Since the $a$ 's and $a^{\dagger}$ 's do not carry units, the newly introduced constants $L$ and $K$ do.

It is reasonable to require the existence of a physical region in which the usual quantum mechanics is recovered as a limiting casef even if $q^{2} \neq 1$. This could be

\footnotetext{
${ }^{1}$ unlike using as usual ordinary complex integration for the bosonic case and Berezin integration for the fermionic case

${ }^{2}$ weakening this restriction, one may generalisable the ansatz Eqs.7
} 
achieved if the commutation relations come out in the form $[x, p]=i \hbar+f(q, x, p)$ i.e. with the central term being $i \hbar$ without any $q$ - factors. The uncertainty relation $\Delta x \Delta p \geq \frac{\hbar}{2}+\frac{1}{2}\langle f(q, x, p)\rangle$ should then reduce to the usual relations where $\langle f\rangle$ is negligible. The actual commutation relations come out as follows:

\subsection{Commutation relations}

We express the commutation relations Eqs.4 in terms of the $x$ 's and $p$ 's:

$$
\begin{aligned}
{\left[x_{r}, p_{r}\right]=} & \frac{4 i L_{r} K_{r}}{q^{2}+1} \\
& +\frac{4 i L_{r} K_{r}\left(q^{2}-1\right)}{q^{2}+1}\left[\sum_{s \leq r}\left(\frac{x_{s}^{2}}{4 L_{s}^{2}}+\frac{p_{s}^{2}}{4 K_{s}^{2}}\right)-\sum_{t<r} \frac{1}{4 i L_{t} K_{t}}\left[x_{t}, p_{t}\right]\right]
\end{aligned}
$$

The commutators $\left[x_{t}, p_{t}\right]$ (with $t<r$ ) can be eliminated by iterating the equation.

Choosing the products $L_{s} K_{s}$ appropriately we can indeed bring the commutation relation into the desired form, i.e. with the central term being exactly $i \hbar$ :

The ansatz

$$
\left[x_{r}, p_{r}\right]=i \hbar+i \sum_{s \leq r} \beta_{s}\left(\frac{x_{s}^{2}}{4 L_{s}^{2}}+\frac{p_{s}^{2}}{4 K_{s}^{2}}\right)
$$

leads for the central term to the equation:

$$
i \hbar=\frac{4 i L_{r} K_{r}}{q^{2}+1}-i \hbar L_{r} K_{r} \frac{q^{2}-1}{q^{2}+1} \sum_{t<r} \frac{1}{L_{t} K_{t}}
$$

Solving this equation we get relations between the $L_{r}$ and $K_{r}$ :

$$
L_{r} K_{r}:=\frac{\hbar}{2}\left(\frac{q^{2}+1}{2}\right)^{r}
$$

From this follows $\beta_{r}$ immediately:

$$
\beta_{r}=4 L_{r} K_{r} \frac{q^{2}-1}{q^{2}+1}=\hbar\left(q^{2}-1\right)\left(\frac{q^{2}+1}{2}\right)^{r-1}
$$

Thus the commutation relations take final form:

$$
\left[x_{r}, p_{r}\right]=i \hbar+i \hbar\left(q^{2}-1\right) \sum_{s \leq r}\left(\frac{q^{2}+1}{2}\right)^{s-1}\left(\frac{x_{s}^{2}}{4 L_{s}^{2}}+\frac{p_{s}^{2}}{4 K_{s}^{2}}\right)
$$

The mixed commutation relations read for $s>r$ :

$$
\left[x_{s}, p_{r}\right]=i \frac{L_{r}}{K_{r}} \frac{q-1}{q+1}\left\{x_{s}, x_{r}\right\}
$$




$$
\left[x_{s}, x_{r}\right]=i \frac{K_{r}}{L_{r}} \frac{q-1}{q+1}\left\{x_{s}, p_{r}\right\}
$$

For $s<r$ one gets:

$$
\begin{gathered}
{\left[x_{s}, p_{r}\right]=i \frac{K_{s}}{L_{s}} \frac{q-1}{q+1}\left\{p_{s}, p_{r}\right\}} \\
{\left[p_{s}, p_{r}\right]=-i \frac{L_{s}}{K_{s}} \frac{q-1}{q+1}\left\{x_{s}, p_{r}\right\}}
\end{gathered}
$$

To see this, solve Eqs.7 for the $a$ 's and $a^{\dagger}$ 's, express Eqs.1궁 in terms of the $x$ 's and $p$ 's and find e.g. Eq.15 from Eq.11 $+(\text { Eq. [1 })^{+}+$Eq.3 $+(\text {Eq.3 })^{+}$. If $q^{2}=1$ the constants $K$ and $L$ drop out of the commutation relations, reflecting that in ordinary quantum mechanics a length or a momentum scale can only be set by the Hamiltonian i.e. by choosing a particular system. Here, for $q^{2} \neq 1$ the $K$ and $L$ appear in the commutation relations, thus these scales become a property of the quantum mechanical formalism itself.

\section{$2.2 \quad$ A maximal set of commuting observables}

The operators $x_{i}$ (as well as the operators $p_{i}$ ) no longer commute among themselves. Thus we conclude that the position operators can not be simultaneously diagonalised and the same for the momentum operators.

Before studying the structure of the noncommutative 'configuration space' and the noncommutative momentum space in more detail, let us mention that for example the following symmetric operators $h_{i}$ can still serve as a set of commuting observables:

$$
h_{i}:=\frac{x_{i}^{2}}{4 L_{i}^{2}}+\frac{p_{i}^{2}}{4 K_{i}^{2}} \quad(r=1, \ldots, n)
$$

The symmetry is obvious. To prove commutativity we use that

$$
\left[a_{i}^{\dagger} a_{i}, a_{j}^{\dagger} a_{j}\right]=0 \quad \forall i, j
$$

which follows immediately from Eqs.1. 3 .

With the definitions Eqs.7 follows

$$
a_{r}^{\dagger} a_{r}=\frac{x_{r}^{2}}{4 L_{r}^{2}}+\frac{p_{r}^{2}}{4 K_{r}^{2}}-\frac{1}{4 i L_{r} K_{r}}\left[x_{r}, p_{r}\right]
$$

Eliminating the commutators using Eq.14 yields

$$
a_{r}^{\dagger} a_{r}=h_{r}-\frac{1}{4 i L_{r} K_{r}}\left(i \hbar+i \hbar\left(q^{2}-1\right) \sum_{s \leq r}\left(\frac{q^{2}+1}{2}\right)^{s-1} h_{s}\right)
$$

which eventually gives

$$
\left[h_{i}, h_{j}\right]=0 \quad \forall i, j
$$

The eigenstates of the operators $h_{i}$ are of course generalised Hermite wave functions. Let us however focus on the operators $x$ and $p$, i.e on configuration and momentum space. 


\section{Uncertainty relation}

For simplicity we first consider the 1 dimensional case where Eq.14 reads:

$$
[x, p]=i \hbar+i \hbar\left(q^{2}-1\right)\left(\frac{x^{2}}{4 L^{2}}+\frac{p^{2}}{4 K^{2}}\right)
$$

with

$$
K=\frac{\hbar}{4 L}\left(q^{2}+1\right)
$$

We will now study the situation for $q^{2}>1$. The case $q^{2}<1$ is quite different and will be discussed elsewhere. The following (standard) derivation of the uncertainty relation holds on every domain $D^{\prime}$ of $x$ and $p$, on which both operators are symmetric and have their images in the domain. The above given domain $D$ is an example.

We start with the trivial statement that the following norm is positive:

$$
|((x-\langle v, x . v\rangle)+i \alpha(p-\langle v, p . v\rangle)) v| \geq 0 \quad \forall v \in D^{\prime} \quad \forall \alpha
$$

Using that $x$ and $p$ are symmetric on $D^{\prime}$ this is for all real $\alpha$ :

$$
(\Delta x)^{2}+\alpha^{2}(\Delta p)^{2}+i \alpha\langle v,[x, p] . v\rangle \geq 0
$$

with the usual definitions, e.g.:

$$
(\Delta x)^{2}:=\left\langle v,(x-\langle v, x . v\rangle)^{2} \cdot v\right\rangle
$$

This can be put into the form:

$$
(\Delta p)^{2}\left(\alpha-\frac{\hbar A}{\alpha(\Delta p)^{2}}\right)^{2}-\frac{\hbar^{2} A^{2}}{4(\Delta p)^{2}}+(\Delta x)^{2} \geq 0
$$

with $A$ coming from the rhs of the commutation relation Eq.24:

$$
A=1+\left(q^{2}-1\right)\left(\frac{(\Delta x)^{2}+\langle x\rangle^{2}}{4 L^{2}}+\frac{(\Delta p)^{2}+\langle p\rangle^{2}}{4 K^{2}}\right)
$$

(we abbreviated e.g. $\langle v, x . v\rangle$ as $\langle x\rangle$ )

Now for any given $v \in D^{\prime}$ we get the most restrictive inequality when choosing $\alpha=\hbar A / 2(\Delta p)^{2}$ so that the first term on the lhs of Eq.28, which can't be negative, vanishes. This yields for all $v$ in $D^{\prime}$ the uncertainty relation

$$
\Delta x \Delta p \geq \frac{\hbar}{2}\left(1+\left(q^{2}-1\right)\left(\frac{(\Delta x)^{2}+\langle x\rangle^{2}}{4 L^{2}}+\frac{(\Delta p)^{2}+\langle p\rangle^{2}}{4 K^{2}}\right)\right)
$$




\subsection{Minimal uncertainties in position and momentum}

In order to analyse the content of this uncertainty relation we express it in 'polar coordinates'

$$
\Delta x:=2 \operatorname{Lr} \cos \alpha \quad \text { and } \quad \Delta p:=2 K r \sin \alpha
$$

where it takes the form:

$$
r^{2} \geq \frac{1+\left(q^{2}-1\right)\left(\frac{\langle x\rangle^{2}}{4 L^{2}}+\frac{\langle p\rangle^{2}}{4 K^{2}}\right)}{\left(q^{2}+1\right) \sin 2 \alpha-\left(q^{2}-1\right)}
$$

with

$$
\sin 2 \alpha>\frac{q^{2}-1}{q^{2}+1}
$$

From Eq. 33 follows that the minimal $\alpha$ is larger than 0 and the maximal $\alpha$ is smaller than $\pi / 2$. Thus the hyperbola of the ordinary uncertainty relation, having the $\Delta x$ and the $\Delta p$ axes as asymptotes has turned into a graph with asymptotes that are no longer parallel to the axes. From Eq. 32 follows that $r$ is always larger than 0 . We thus conclude that there are minimal nonzero uncertainties in the positions and the momenta.

In order to calculate them we define

$$
f(\Delta x, \Delta p):=\Delta x \Delta p-\frac{\hbar}{2}\left(1+\left(q^{2}-1\right)\left(\frac{(\Delta x)^{2}+\langle x\rangle^{2}}{4 L^{2}}+\frac{(\Delta p)^{2}+\langle p\rangle^{2}}{4 K^{2}}\right)\right)
$$

and find e.g. $\Delta x_{\min }$ by solving

$$
\frac{\partial}{\partial \Delta p} f(\Delta x, \Delta p)=0 \quad \text { and } \quad f(\Delta x, \Delta p)=0
$$

which has the solution:

$$
\left(\Delta x_{\min }\right)^{2}=L^{2} \frac{q^{2}-1}{q^{2}}\left(1+\left(q^{2}-1\right)\left(\frac{\langle x\rangle^{2}}{4 L^{2}}+\frac{\langle p\rangle^{2}}{4 K^{2}}\right)\right)
$$

Thus the absolutely smallest uncertainty in the position is:

$$
\Delta x_{0}=L \sqrt{\frac{q^{2}-1}{q^{2}}}
$$

Analogously one obtains the absolutely smallest uncertainty in the momentum:

$$
\Delta p_{0}=K \sqrt{\frac{q^{2}-1}{q^{2}}}
$$


Due to Eq.25 there were two free parameters: The length $L$ and $q$. Instead we can now use $\Delta x_{0}$ and $\Delta p_{0}$ as the free parameters and express $L, K$ and $q$ in terms of these:

$$
\begin{gathered}
L=\Delta x_{0} \sqrt{\frac{2 \Delta x_{0} \Delta p_{0}+\hbar+\sqrt{4\left(\Delta x_{0}\right)^{2}\left(\Delta p_{0}\right)^{2}+(\hbar)^{2}}}{4 \Delta x_{0} \Delta p_{0}}} \\
K=\Delta p_{0} \sqrt{\frac{2 \Delta x_{0} \Delta p_{0}+\hbar+\sqrt{4\left(\Delta x_{0}\right)^{2}\left(\Delta p_{0}\right)^{2}+(\hbar)^{2}}}{4 \Delta x_{0} \Delta p_{0}}} \\
q=\sqrt{\left(2 \Delta x_{0} \Delta p_{0}+\sqrt{4\left(\Delta x_{0}\right)^{2}\left(\Delta p_{0}\right)^{2}+\hbar^{2}}\right) / \hbar}
\end{gathered}
$$

The commutation relation Eq.24 then takes the form:

$$
[x, p]=i \hbar+i g\left(\Delta x_{0}, \Delta p_{0}\right)\left(\frac{x^{2}}{\left(\Delta x_{0}\right)^{2}}+\frac{p^{2}}{\left(\Delta p_{0}\right)^{2}}\right)
$$

where

$$
g\left(\Delta x_{0}, \Delta p_{0}\right):=4 \frac{\Delta x_{0} \Delta p_{0}}{\hbar} \frac{2 \Delta x_{0} \Delta p_{0}+\sqrt{4\left(\Delta x_{0} \Delta p_{0}\right)^{2}+\hbar^{2}}-\hbar}{2 \Delta x_{0} \Delta p_{0}+\sqrt{4\left(\Delta x_{0} \Delta p_{0}\right)^{2}+\hbar^{2}}+\hbar}
$$

Let us now identify the physical region where the ordinary quantum mechanical behaviour is recovered:

Since physically we know that $\Delta x_{0}$ and $\Delta p_{0}$ can only be very small, say $\Delta x_{0} \Delta p_{0} \ll$ $\hbar / 2$, we expand $g$ to the first nonzero order in this product and arrive at the simplified commutation relation:

$$
[x, p]=i \hbar+\frac{4 i}{\hbar}\left(x^{2}\left(\Delta p_{0}\right)^{2}+p^{2}\left(\Delta x_{0}\right)^{2}\right)
$$

Now it becomes clear how in our formalism not only the behaviour for small distances and momenta is altered: Also for expectation values of $x^{2}$ or $p^{2}$ large enough to make the second term on the rhs of the order $\hbar$ or larger, the behaviour will be significantly changed. The region of approximately ordinary quantum mechanical behaviour is thus specified through:

$$
\begin{aligned}
& \left(\Delta x_{0}\right)^{2} \ll x^{2} \ll \frac{\hbar^{2}}{4\left(\Delta p_{0}\right)^{2}} \\
& \left(\Delta p_{0}\right)^{2} \ll p^{2} \ll \frac{\hbar^{2}}{4\left(\Delta x_{0}\right)^{2}}
\end{aligned}
$$

From the point of view of wave-particle dualism, meaning e.g. that high momenta are needed to measure small distances, this is of course a reasonable result. 


\subsection{Generalisation to $n$ dimensions}

Let us now return to the $n$ dimensional case where we will recover essentially the same behaviour:

It is straightforward to see that the derivation of Eq.30 also works in the $n$ dimensional case i.e. we get:

$$
\Delta x_{j} \Delta p_{j} \geq \frac{1}{2}\left|\left\langle\left[x_{j}, p_{j}\right]\right\rangle\right|
$$

where the commutator is given in Eq.14. There are again minimal uncertainties $\Delta x_{i_{\text {min }}}$ and $\Delta p_{i_{\text {min }}}$ which are calculated using

$$
f\left(\Delta x_{j}, \Delta p_{j}\right):=\Delta x_{j} \Delta p_{j}-\frac{1}{2}\left|\left\langle\left[x_{j}, p_{j}\right]\right\rangle\right|
$$

and solving, in order to obtain e.g. $\Delta x_{j_{\min }}$ :

$$
\frac{\partial}{\partial \Delta p_{j}} f\left(\Delta x_{j}, \Delta p_{j}\right) \quad \text { and } \quad f\left(\Delta x_{j}, \Delta p_{j}\right)=0
$$

The solutions are:

$\Delta x_{j_{\text {min }}}=L_{j} \frac{q^{2}-1}{q^{2}}\left(\frac{q^{2}+1}{2}\right)^{1-j} \cdot s(j) \quad$ and $\quad \Delta p_{j_{m i n}}=K_{j} \frac{q^{2}-1}{q^{2}}\left(\frac{q^{2}+1}{2}\right)^{1-j} \cdot s(j)$

with

$$
\begin{aligned}
s(j)=1+ & \left(q^{2}-1\right) \sum_{s<j}\left(\frac{q^{2}+1}{2}\right)^{s-1}\left(\frac{\left\langle x_{s}^{2}\right\rangle}{4 L_{s}^{2}}+\frac{\left\langle p_{s}^{2}\right\rangle}{4 K_{s}^{2}}\right) \\
& +\left(q^{2}-1\right)\left(\frac{q^{2}+1}{2}\right)^{j-1}\left(\frac{\left\langle x_{j}\right\rangle^{2}}{4 L_{j}^{2}}+\frac{\left\langle p_{j}\right\rangle^{2}}{4 K_{j}^{2}}\right)
\end{aligned}
$$

Due to Eq.12 the $K_{j}$ and the $L_{j}$ are not independed, so that we are left with in total $n+1$ free parameters. One may choose them to be e.g. $q$ and the $n$ minimal uncertainties in the positions.

It would now of course be interesting to study whether symmetric operators of angular momentum can be expressed in terms of the position and momentum operators and how they reflect the appearance of minimal uncertainties. Let us however first study the functional analysis of the position and momentum operators in more detail.

\section{$4 \quad$ Functional analysis of $x$ and $p$}

We first consider the one dimensional case. The above derived uncertainty relation Eq. 30 holds on every domain $D^{\prime}$ on which both, $x$ and $p$ are symmetric and have their 
ranges in $D^{\prime}$. The uncertainty relation implied minimal nonzero uncertainties in the positions and momenta.

Now if there was a $v_{\lambda} \in D^{\prime}$ that is eigenvector e.g. of $x$ :

$$
x . v_{\lambda}=\lambda v_{\lambda}
$$

one would then of course have

$$
(\Delta x)^{2}=\left\langle v_{\lambda}\left|\left(x-\left\langle v_{\lambda}, x \cdot v_{\lambda}\right\rangle\right)^{2}\right| v_{\lambda}\right\rangle=0
$$

which would be a contradiction. We thus conclude that there is no domain on which $x$ and $p$ are symmetric and have eigenvectors. Let us now study the functional analysis of $x$ in more detail, the analysis for $p$ is completely analogous.

\subsection{The operators $x, x^{*}$ and $x^{* *}$}

We start be choosing for $x$ the domain $D_{x}:=D$ (the finite linear combinations of the vectors $\left(a^{\dagger}\right)^{r}|0\rangle$ with $\left.r=0,1,2, \ldots\right)$, on which $x$ and $p$ are obviously symmetric and have their image in $D_{x}$. We can thus already conclude from above that $x$ has no eigenvectors in $D_{x}$. Indeed, the eigenvalue problem

$$
x \cdot v_{\lambda}=\lambda v_{\lambda} \quad \text { with } \quad v_{\lambda}=\sum_{r=0}^{\infty} f_{r}(\lambda) \frac{\left(a^{\dagger}\right)^{r}}{\sqrt{[r]_{q} !}}|0\rangle
$$

can be solved for all complex $\lambda$, but from the recursion formula that we obtain for the coefficients $f_{r}(\lambda)$ of $v_{\lambda}$ it is clear that infinitely many of them are nonzero, thus $v_{\lambda} \notin D_{x}$ : Explicitely, in the orthonormalised basis

$$
e_{r}:=\frac{\left(a^{\dagger}\right)^{r}}{\sqrt{[r]_{q} !}}|0\rangle
$$

the matrix elements of $x$ are

$$
x_{r s}=L\left(\sqrt{[r]_{q}} \delta_{r, s+1}+\sqrt{[s]_{q}} \delta_{r+1, s}\right)
$$

and the recursion formula that we obtain for the coefficients $f_{r}(\lambda)$ of the vector $v_{\lambda}$ thus reads:

$$
\sqrt{[r+1]_{q}} f_{r+1}=\frac{\lambda}{L} f_{r}-\sqrt{[r]_{q}} f_{r-1}
$$

Let us now consider the adjoint $x^{*}$ of $x$. It is a closed operator since $\bar{D}_{x}=\mathcal{H}$ and has the domain:

$$
D_{x^{*}}=\left\{v \in \mathcal{H} \mid \quad \exists w \in \mathcal{H} \quad \forall a \in D_{x}: \quad\langle v, x . a\rangle=\langle w, a\rangle\right\}
$$

Of course $D_{x} \subset D_{x^{*}}$ and, using the above mentioned recursion formula, one proves that actually all $v_{\lambda}$ are normalisable and are contained in the domain $D_{x^{*}}$ : 
To see this we rewrite the recursion formula in matrix form:

$$
\left(\begin{array}{c}
f_{r+1} \\
f_{r}
\end{array}\right)=\frac{1}{\sqrt{[r]_{q}[r+1]_{q}}}\left(\begin{array}{cc}
\frac{\lambda^{2}}{L^{2}}-[r]_{q}, & -\frac{\lambda}{L} \sqrt{[r-1]_{q}} \\
\frac{\lambda}{L} \sqrt{[r+1]_{q}}, & -\sqrt{[r-1]_{q}[r+1]_{q}}
\end{array}\right)\left(\begin{array}{c}
f_{r-1} \\
f_{r-2}
\end{array}\right)
$$

The iteration matrix simplifies for large $r$ to

$$
\left(\begin{array}{cc}
-\sqrt{[r]_{q} /[r+1]_{q}} & 0 \\
0 & -\sqrt{[r-1]_{q} /[r]_{q}}
\end{array}\right)
$$

and eventually goes like

$$
\left(\begin{array}{cc}
-1 /|q| & 0 \\
0 & -1 /|q|
\end{array}\right)
$$

Since $q^{2}>1$ we can thus apply the quotient criterium (behaviour like a geometrical series) to conclude that

$$
\sum_{r=0}^{\infty} f_{r}^{*}(\lambda) f_{r}(\lambda)<\infty
$$

i.e. that all $v_{\lambda}$ are normalisable ? They are obviously contained in $D_{x^{*}}$ and are thus eigenvectors of $x^{*}$. Since there are nonreal eigenvalues we conclude that $x^{*}$ is not symmetric. This allows its eigenvectors to be linearily dependend. They are actually in general linearily dependend on each other since the Hilbert space $\mathcal{H}$ is seperable and there is an uncountable infinite number of eigenvectors $v_{\lambda}$. An analytic expression for the scalar product of two normalised eigenvectors $\left\langle\hat{v}_{\lambda}, \hat{v}_{\lambda^{\prime}}\right\rangle$ has not yet been worked out. However, the numerical approximation converges as quickly as a geometrical series.

The operator $x^{* *}$ is much better behaved than $x^{*}$, since it is closed and symmetric, as every bi-adjoint of a densly defined symmetric operator.

Its domain

$$
D_{x^{* *}}=\left\{v \in \mathcal{H} \mid \quad \exists w \in \mathcal{H} \quad \forall a \in D_{x^{*}}: \quad\left\langle v, x^{*} . a\right\rangle=\langle w, a\rangle\right\}
$$

is in between those of $x$ and $x^{*}: D_{x} \subset D_{x^{* *}} \subset D_{x^{*}}$ and it does not contain any eigenvectors $v_{\lambda}$.

\subsection{Self-adjoint extensions}

We now apply the standard procedure, see e.g. [18, 19] for checking for self-adjoint extensions of closed symmetric operators:

\footnotetext{
${ }^{3}$ It also implies that there are no normalisable eigenvectors when $q^{2}<1$

${ }^{4}$ Note that [18] defines 'hermitean' as synonymous to self-adjoint while [19] uses it as synonymous to symmetric.
} 
The idea is to check whether the 'Cayley transform' can be isometrically extended. An inverse Cayley transform then yields a self-adjoint extension of $x^{* *}$. To this end we calculate the orthogonal complement of the spaces:

$$
L_{ \pm i, x^{* *}}:=\left(x^{* *} \pm i\right) \cdot D_{x^{* *}}
$$

Since $x^{* *}$ is closed, symmetric and has $\bar{D}_{x^{* *}}=\mathcal{H}$, these deficiency subspaces $L_{ \pm i, x^{* *}}^{\perp}$ can be written as

$$
L_{ \pm i, x^{* *}}^{\perp}:=\operatorname{ker}\left(x^{*} \mp i\right) \cdot D_{x^{*}}
$$

Here we used that $x^{* * *}=x^{*}$ which holds because $x^{*}$ is closed and $\bar{D}_{x^{*}}=\mathcal{H}$. Since there is only one $v_{i}$ and one $v_{-i}$ the dimensions of these spaces, i.e. the deficiency indices are both equal to 1 . We can thus define the following one-parameter family of self-adjoint extensions:

$$
x_{s a}(\phi) \cdot a:=i(b+U . b) \quad \text { for all } \quad a=b-U . b
$$

with the isometric operator $U$ defined on $\left(x^{* *}+i\right) \cdot D_{x^{* *}} \oplus \mathbf{C} v_{i}$ as

$$
U . v:=\left(x^{* *}-i\right)\left(x^{* *}+i\right)^{-1} \cdot v \quad \forall v \in\left(x^{* *}+i\right) . D_{x^{* *}}=L_{+i, x^{* *}}
$$

and

$$
U . \alpha v_{i}:=\alpha e^{i \phi} v_{-i}
$$

Here $\phi$ is a free real parameter, labeling the self-adjoint extensions. For the eigenvalues one can stay with the extended Cayley transform $U$, calculate its eigenvalues, and an inverse Möbius transform then maps them onto the eigenvalues of $x_{s a}(\phi)$.

The analysis for $p$ analogously leads to a one-parameter family of self-adjoint extensions $p_{s a}(\psi)$. One may now be tempted to try to fix the choice of the extension parameters $\phi$ and $\psi$ by requiring that $x_{s a}(\phi)$ and $p_{s a}(\psi)$ be defined on the same domain. One would then like to diagonalise $x_{s a}(\phi)$ to obtain a coordinate space representation or to diagonalise $p_{s a}(\psi)$ to obtain a momentum space representation.

However, we know from section 3 that $x$ and $p$ cannot be extended to a common domain on which they have eigenvectors.

\subsection{The $n$ dimensional case}

One finds in the $n$ dimensional case essentially the same situation:

We calculate the matrix elements of e.g. the position operator in $j$-direction $x^{j}$ in the orthonormal basis of the vectors:

$$
e_{s_{1}, \ldots, s_{n}}:=\frac{\left(a_{1}^{\dagger}\right)^{s_{1}} \cdot \ldots \cdot\left(a_{n}^{\dagger}\right)^{s_{n}}}{\sqrt{\left[s_{1}\right]_{q} ! \cdot \ldots \cdot\left[s_{n}\right]_{q} !}}|0\rangle
$$


Using Eqs.11-1 one finds the matrix elements:

$$
\begin{aligned}
x_{r_{1}, \ldots, r_{n}, s_{1}, \ldots, s_{n}}^{j}=\quad & L_{j}\left(\sqrt{\left[r_{j}\right]_{q}} \delta_{r_{j}, s_{j}+1}+\sqrt{\left[s_{j}\right]_{q}} \delta_{r_{j}+1, s_{j}}\right) \cdot \\
& q^{r_{1}+\ldots+r_{j-1}} \delta_{r_{1}, s_{1}} \cdot \ldots \cdot \delta_{r_{j-1}, s_{j-1}} \delta_{r_{j}+1, s_{j}+1} \cdot \ldots \cdot \delta_{r_{n}, s_{n}}
\end{aligned}
$$

The eigenvalue problem

$$
x_{j} v_{\lambda}=\lambda v_{\lambda}
$$

is then solved by all

$$
v_{\lambda}=\sum_{s_{j}=0}^{\infty} f_{s_{1}, \ldots, s_{j}, \ldots, s_{n}}(\lambda) \quad e_{s_{1}, \ldots, e_{n}}
$$

with coefficients that obey the recursion formula (all $s_{i}$ are kept fixed except $s_{j}$ ):

$$
\begin{aligned}
\sqrt{\left[r_{j}+1\right]_{q}} f_{s_{1}, \ldots, s_{j}+1, \ldots, s_{n}}(\lambda)= & \frac{\lambda}{L} q^{-\left(r_{1}+\ldots+r_{j-1}\right)} f_{s_{1}, \ldots, s_{j}, \ldots, s_{n}}(\lambda) \\
& -\sqrt{\left[r_{j}\right]_{q}} f_{s_{1}, \ldots, s_{j}-1, \ldots, s_{n}}(\lambda)
\end{aligned}
$$

The scaling factor $q^{-\left(r_{1}+\ldots+r_{j-1}\right)}$ implies that $x_{j}$ 'sees' not only its own direction, reflecting that the position operators no loeger commute (Eq.16). Nevertheless the same arguments as for the one dimensional case go through again and we conclude that $v_{\lambda}$ is normalisable for all complex $\lambda$. Again the operators $x_{k}^{*}$ are nonsymmetric while the operators $x_{k}^{* *}$ are symmetric and closed. There is however a new feature which is that the deficiency subspaces are now much larger than in the one dimensional case because the operators $x_{k}^{*}$ now have countable infinitely many linearly independend eigenvectors $v_{i}$ and $v_{-i}$. Since both deficiency subspaces are of the same size, $x_{k}$ has self-adjoint extensions. The same arguments apply for the momentum operators $p_{k}$. However, we conclude again from the uncertainty relation that there is no common domain on which all these operators are symmetric and have eigenvectors.

\section{$5 \quad$ Summary and Outlook}

We thus arrive at the following picture:

While in classical mechanics the states can have exact positions and momenta, in quantum mechanics there is the well known uncertainty principle, not allowing $x$ and $p$ to have common eigenvectors. Nevertheless $x$ and $p$ seperately do have 'eigenvectors', though non-normalisable ones. The spectrum is continuous, namely the whole configuration or momentum space.

From the above discussion we conclude that the 'noncommutative geometry'- or quantum group generalisation of the Heisenberg algebra has further consequences for the $x$ and $p$ : It is not only that the $x$ and $p$ have no common eigenvectors, here they even have no common domain on which they (are symmetric and) have eigenvectors. The $x$ and $p$ seperately do have (infinitely many) self-adjoint extensions and 
all candidates for eigenvectors are normalisable. Thus the spectra of the self-adjoint extensions of the position and the momentum operators are no longer continuous. Only discrete ('lattices' of) eigenvalues can occur.

In general one will however represent the $x$ and $p$, i.e. the full Heisenberg algebra, on a common domain on which the $x$ and $p$ are symmetric. The symmetry is of course to insure that all physical expectation values are real. We concluded that in this case the $x$ and $p$ cannot have eigenvectors. The non-existence of position or momentum eigenvectors meant of course the non-existence of absolute precision in position or momentum measurements. We found minimal nonzero uncertainties in these measurements. The maximal common domain on which the $x$ and $p$ are symmetric remains to be determined.

It should be interesting to examine whether this quantum mechanical formalism with 'built in' minimal uncertainties in the $x$ and $p$ can find applications in effective theories, where minimal nonzero uncertainties in position or momentum measurements appear naturally, e.g. in solid state or nuclear physics.

The present paper also supports the idea that noncommtutive geometry mathe-

matics has the potential to regularise the small $\Delta x$ i.e. the ultraviolet, as well as the small $\Delta p$, i.e. the infrared behaviour of quantum field theories.

\section{Acknowledgements}

I would like to thank J.Mickelson and J.Wess for their interest and useful critisims.

\section{References}

[1] V.G.Drinfel'd, in Proc. ICM Berkeley, AMS, Vol. 1 (1986) 798-820

[2] L.D.Faddeev, N.Yu.Reshetikhin, L.A.Takhtajan, Alg.Anal. 1, 1, (1989) 178

[3] S.Majid, Int. J. Mod. Phys. A. Vol. 5, No 1, (1990) 1-91

[4] A.Connes, Publ. I.H.E.S. 62 (1986) 257

[5] A.Kempf, to appear in Proc. XXII DGM Conference, Sept. '93, Ixtapa, Mexiko

[6] A.Kempf, Lett. Math. Phys. 26: (1992) 1-12

[7] W.Pusz, S.Woronowicz, Rep. Math. Phys. 27 (1989) 231

[8] J.Wess, B.Zumino, Nucl. Phys. Proc. Suppl. 18 B (1991) 302

[9] S.Majid, J. Math. Phys. 34 (10) (1993) 4843-4856

[10] A.Macfarlane, J. Phys. A 22 (1989) 4581

[11] L.Biedenharn, J. Phys. A 22 (1989) L 873 
[12] J.Schwenck, J.Wess, Phys. Lett. B 291, (1992) 273

[13] A.Hebecker, W.Weich, in Proc. XIX Symposium Ahrenshoop, Eds. B.Dörfel, E.Wieczorek, DESY Preprint 93-013, (1992)

[14] A.Kempf, in Proc. XX DGM- Conference, June '91, New York, Eds. S.Catto, A.Rocha, World Scientific, (1991) 546

[15] A.Kempf, in Proc. XIX Symposium Ahrenshoop in Wendisch-Rietz, Eds. B.Dörfel, E.Wieczorek, DESY Preprint 93-013, (1992)

[16] L.Baulieu, E.G.Floratos, Phys. Lett. B 258 (1991) 171

[17] A.Kempf, J. Math. Phys., Vol. 34, No.3, (1993) 969-987

[18] F.Hirzebruch, W.Scharlau, 'Einf. Funktionalanalysis', B.I. (1971)

[19] N.I. Akhiezer, I.M. Glazman, 'Theory of Lin. Op. in Hilbert space', F. Ungar Publ. (1963) 